\title{
Synthesis, characterisation and catalytic activity of dithiocarbazate Schiff base complexes in oxidation of cyclohexane
}

\begin{abstract}
Schiff base complexes of transition metals can be used for catalytic oxidation of hydrocarbons in the presence of tert-butyl hydroperoxide (TBHP). In this work, metal complexes of dithiocarbazate Schiff bases (abbreviated as NiSBdiAP, CuSBdiAP, CoSBdiAP, FeSBdiAP, MnSBdiAP and ZnSBdiAP, in which SBdiAP represents the Schiff base) were synthesized and characterized using several techniques; Fourier transforms infrared spectroscopy, nuclear magnetic resonance, $1 \mathrm{H}$ NMR and 13C NMR, magnetic susceptibility measurements, molar conductivity, ultraviolet-visible spectroscopy, and inductively coupled plasma spectrometry. The oxidation of cyclohexane by the prepared metal complexes as catalysts was investigated in the presence of TBHP as an oxidative source under mild conditions. The product was analyzed using gas chromatography. It was found that cyclohexanol and cyclohexanone were the main products of the oxidation reaction. The iron complex of Schiff base derived from 2,6-diacetyl pyridine and $S$ benzyldithiocarbazate showed the highest activity during the screening studies towards cyclohexane oxidation. The results showed that the time of the reaction, temperature, and the concentration of TBHP and the catalyst type influenced the selectivity and conversion of cyclohexane oxidation. The selectivity of the reaction was $96 \%$ indicating the significance of the work toward industrial oxidation of cyclohexane.
\end{abstract}

Keyword: Oxidation; Dithiocarbazate Schiff bases; Metal complexes; Selectivity 